\title{
AN ALTERNATE CHARACTERIZATION OF CONTINUITY
}

\author{
BRUCE D. SMITH
}

ABSTRACT. The notion of an almost continuous function has previously been defined in two different ways. This paper explores the relationship between these two notions, leading to an alternate characterization of continuity for real functions.

Introduction. The notion of an almost continuous function has been defined in two different ways by $\mathbf{J}$. Stallings and by $\mathrm{T}$. Husain. In their paper, Properties of almost continuous functions, Paul E. Long and Earl E. $\mathrm{McGehee,} \mathrm{Jr}$, have raised the question of the relationship between these two notions [1, p. 180]. The study of this question has led to an alternate characterization of continuity for real functions, which is developed in this paper. I extend my thanks to John W. Lamperti for his many helpful suggestions.

Throughout the following, let $G(f) \subset X \times Y$ denote the graph of the function $f: X \rightarrow Y$, and let $R$ denote the reals with the standard topology. Stallings defines almost continuity as follows [2, p. 252]:

Definition 1. A function $f: X \rightarrow Y$, where $X$ and $Y$ are topological spaces, is almost continuous if and only if for any open set $N \subset X \times Y$, if $G(f) \subset N$, there exists a continuous function $g: X \rightarrow Y$ such that $G(g) \subset N$.

Husain defines almost continuity somewhat differently in [3]:

Definition 2. A function $f: X \rightarrow Y$, where $X$ and $Y$ are topological spaces, is almost continuous at $x \in X$ if and only if for each open set $V \subset Y$ containing $f(x), \mathrm{Cl}\left(f^{-1}(V)\right)$ is a neighborhood of $x$. If $f$ is almost continuous at each point of $X$, then $f$ is called almost continuous.

Clearly any continuous function satisfies both of these definitions. There are, however, many functions which satisfy one or the other of these definitions of almost continuity, and yet are not continuous. Examples of such functions are included in this paper.

Results. It is useful in what follows to introduce a third class of functions. We define these as follows:

Definition 3. A function $f: X \rightarrow Y$, where $X$ and $Y$ are topological spaces, is of the Cesàro type if and only if there exist nonempty open sets $U \subset X$ and $V \subset Y$ such that, for all $y \in V, U \subset \mathrm{Cl}\left(f^{-1}(y)\right)$.

Received by the editors September 21, 1972.

AMS (MOS) subject classifications (1970). Primary 26A15, 54C05, 54C10, 54C30.

Key words and phrases. Continuous, almost continuous, Cesàro type, connected graph.

(c) American Mathematical Society 1973 
The following example of such a function is due to Cesàro:

EXAMPLE 1. Let $f: R \rightarrow R$ be given by $f(x)=0$ if $x \notin[0,1]$, and $f(x)=$ $\lim \sup \left(a_{1}+a_{2}+\cdots+a_{n}\right) / n$ for $x \in[0,1]$, where the $a_{i}$ are given by the unique nonterminating binary expansion of the number $x=\left(0 . a_{1} a_{2} \cdots\right)$.

Note that this function takes on every value between 0 and 1 on every interval $I \subset[0,1]$. To clarify the relationship between continuous functions and functions of the Cesàro type, we have the following:

LEMMA 1. Any function of the Cesàro type into a connected nontrivial $T_{1}$-space is discontinuous.

Proof. Given a function $f: X \rightarrow Y$ of the Cesàro type, where $X$ is a topological space and $Y$ is a connected nontrivial $T_{1}$-space, there exist open sets $U$ and $V$ as described in Definition 3. A point in a connected nontrivial $T_{1}$-space cannot be open, so $V$ must then contain at least two distinct points, $y_{1}$ and $y_{2}$. Then $V \backslash\left\{y_{2}\right\}$ is an open set containing $y_{1}$. If $f$ is continuous, then $f^{-1}\left(V \backslash\left\{y_{2}\right\}\right)$ is an open set, and $U \cap f^{-1}\left(V \backslash\left\{y_{2}\right\}\right)$ is a nonempty open set. But this is impossible, since $f^{-1}\left(y_{2}\right)$ is dense in $U$. Thus $f$ is discontinuous.

As a further preliminary we note the following, which is proved by Stallings in [2, p. 260]:

Lemma 2. Let $X$ and $Y$ be topological spaces, where $X$ is connected and $X \times Y$ is a completely normal Hausdorff space. If the function $f: X \rightarrow Y$ is almost continuous in the sense of Stallings, then $G(f)$ is connected.

We can now characterize continuity of real functions as follows:

THEOREM. A real function $f: R \rightarrow R$ is continuous if and only if it satisfies the following three conditions:

(i) It is almost continuous in the sense of Stallings.

(ii) It is almost continuous in the sense of Husain.

(iii) It is not of the Cesàro type.

Proof. Necessity. (i) and (ii) are obvious. (iii) follows from Lemma 1.

Sufficiency. Suppose $f$ is not continuous. Choose a point of discontinuity $x_{0}$. There exists an open interval $J=\{y \mid s<y<t\}$ where $f\left(x_{0}\right) \in J$, such that for any open set $N$ containing $x_{0}$ there exists some $w \in N$ with $f(w) \notin J$. Now choose any $s^{\prime}, t^{\prime}$ such that $s<s^{\prime}<f\left(x_{0}\right)<t^{\prime}<t$. Let $J^{\prime}=\left\{y \mid s^{\prime}<y<t^{\prime}\right\}$. By property (ii), there exists an open set $N$ such that $x_{0} \in N$ and $f^{-1}\left(J^{\prime}\right)$ is dense in $N$. But there exists some $w \in N$ such that $f(w) \notin J$. Thus there exists an open interval $K=\{y \mid q<y<r\}$ where $f(w) \in K$ and $\mathrm{Cl}(K) \cap \mathrm{Cl}\left(J^{\prime}\right)=\varnothing$. We may assume without loss of generality that $s^{\prime}<t^{\prime}<q<r$. By property (ii), there exists an open set $M$ such that $w \in M$ and $f^{-1}(K)$ is dense in $M$. Consider the open sets $U=N \cap M$ and $V=$ $\left\{y \mid t^{\prime}<y<q\right\}$. Since $w \in N, M$ we have $U \neq \varnothing$. Clearly $V \neq \varnothing$. Thus property 
(iii) applies, and there exists some $y_{0} \in V$ and some open interval $Q \subset U$ such that $y_{0} \notin f(Q)$. Since $f^{-1}\left(J^{\prime}\right)$ is dense in $N$, we may choose $a, b \in Q$ such that $f(a), f(b) \in J^{\prime}$ and $a<b$. Let $P=\left\{(x, y) \mid a<x<b\right.$ and $\left.y>y_{0}\right\}$. Since $f^{-1}(K)$ is dense in $M$, the set $P \cap G(f)$ is nonempty. By construction, $\operatorname{Bd}(P) \cap G(f)=\varnothing$. Hence $P$ and the complement of its closure are a separation of $G(f)$. But by property (i) and Lemma $2, G(f)$ is connected. Hence $f$ is continuous, and the proof is complete.

It is interesting now to ask the question whether or not the three conditions cited in the theorem are redundant. A partial answer may be obtained by looking at some examples.

EXAMPLE 2. Let $f: R \rightarrow R$ be given by $f(x)=x$ if $x$ is rational and $f(x)=$ $-x$ if $x$ is irrational.

This is a discontinuous function satisfying properties (ii) and (iii), but not (i). Hence property (i) is not redundant and must be included.

EXAMPLE 3. Let $f: R \rightarrow R$ be given by $f(x)=\sin (1 / x)$ for $x \neq 0$, and $f(0)=0$.

This is a discontinuous function satisfying properties (i) and (iii), but not (ii). Hence property (ii) must also be included.

We now consider Example 1, as presented earlier. The graph of this function has been shown to be connected [4, p. 173]. It clearly does not satisfy property (iii), but it does satisfy (ii). Thus, if the converse to Lemma 2 holds, it satisfies (i) as well, and none of the properties is redundant.

Stallings has posed the question of whether such a converse exists [2, p. 261], and it seems a reasonable conjecture that it does. This is a topic for further study.

\section{BIBLIOGRAPHY}

1. Paul E. Long and Earl E. McGehee, Jr., Properties of almost continuous functions, Proc. Amer. Math. Soc. 24 (1970), 175-180. MR 40 \#4931.

2. J. Stallings, Fixed point theorems for connectivity maps, Fund. Math. 47 (1959), 249-263. MR 22 \#8485.

3. T. Husain, Almost continuous mappings, Prace. Mat. 10 (1966), 1-7. MR 36 $\# 3322$.

4. L. Vietoris, Stetige Mengen, Monatsh. Math. Phys. 31 (1921).

Department of Mathematics, University of Virginia, Charlottesville, Virginia 22904 